\title{
Marine fish larvae consistently use external cues for orientation
}

\section{Igal Berenshtein}

University of Miami Cooperative Institute for Marine https://orcid.org/0000-0002-1194-0212

\section{Robin Faillettaz}

University of Miami Cooperative Institute for Marine

Jean-Olivier Irisson

UPMC Univ Paris 06, CNRS

\section{Moshe Kiflawi}

Ben-Gurion University of the NegevUniversity of Tasmania, Hobart

\section{Ulrike Siebeck}

University of Queensland

Jeffery Leis

University of Tasmania

Claire Paris ( $\sim$ cparis@rsmas.miami.edu )

University of Miami Cooperative Institute for Marine

\section{Article}

Keywords: marine fish larvae, larval dispersal, orientation, random walk, cues, meta-analysis

Posted Date: March 10th, 2021

DOI: https://doi.org/10.21203/rs.3.rs-275276/v1

License: (c) (i) This work is licensed under a Creative Commons Attribution 4.0 International License.

Read Full License 


\title{
Marine fish larvae consistently use external cues for orientation
}

Igal Berenshtein ${ }^{\mathrm{a}, \mathrm{b}}$, Robin Faillettaz ${ }^{\mathrm{a}, \mathrm{c}, \mathrm{d}}$, Jean-Oliver Irisson $^{\mathrm{c}}$, Moshe Kiflawi ${ }^{\mathrm{e}, \mathrm{f}}$, Ulrike E. Siebeck $^{\mathrm{g}}$, Jeffery M. Leis ${ }^{\mathrm{h}, \mathrm{i}}$, Claire B. Paris ${ }^{\mathrm{a} *}$

${ }^{a}$ Rosenstiel School of Marine and Atmospheric Science University of Miami 4600 Rickenbacker Causeway, Miami, Florida 33149, United-States

${ }^{b}$ Cooperative Institute for Marine and Atmospheric Studies, Rosenstiel School of Marine and Atmospheric Science, University of Miami, 4600 Rickenbacker Causeway, Miami, FL 33149, USA

${ }^{c}$ Centre National de la Recherche Scientifique, Laboratoire d'Océanographie de Villefranche-sur-Mer (LOV), Sorbonne Universités, UPMC University Paris 06, Villefranche-sur-Mer, France

${ }^{d}$ Ifremer, STH, F-56100 Lorient, France

${ }^{e}$ Department of Life-Sciences, Ben-Gurion University of the Negev, POB 653, 84105 Beer-Sheva, Israel

${ }^{f}$ The Interuniversity Institute for Marine Sciences of Eilat, 88103, Israel

${ }^{g}$ Laboratory for Visual Neuroethology, School of Biomedical Sciences, University of Queensland, St Lucia, QLD 4072, Australia

${ }^{h}$ Ecology and Biodiversity Centre, Institute for Marine and Antarctic Studies, University of Tasmania, Hobart, TAS, 7007, Australia

iIchthyology, Australian Museum Research Institute, Sydney, NSW, 2001, Australia

*Corresponding author: cparis@ rsmas.miami.edu

Keywords: marine fish larvae, larval dispersal, orientation, random walk, cues, meta-analysis

\begin{abstract}
The larval stage is the main dispersive mechanism of most marine teleost fish species. The degree to which larval behavior controls dispersal outcome has been a subject of debate in the past decades. Multiple studies demonstrated orientation mechanisms in several species separately, however a cross-species analysis examining fundamental orientation traits has not been carried out. Here, we apply a cross-species meta-analysis, focusing on the fundamental question of whether larval fish use external cues for directional movement. We compare the observed directional patterns to those expected under a strict use of internal cues. We find that the bulk of fish larvae use external cues for directional swimming, highlighting the contribution of larval orientation behavior to larval dispersal outcome. This finding is an essential step towards a proper implementation of larval behavior in biophysical dispersal models, improving our understanding of population connectivity, and facilitating sustainable management and conservation of marine resources.
\end{abstract}




\section{Main}

The larval phase is the main dispersive stage of most demersal teleost marine fishes, controlling population dynamics and shaping connectivity patterns. As such, it plays a key role in largescale ecological processes such as gene flow and biogeography ${ }^{1}$. The dispersal of larval fish is governed by two main mechanisms, ocean currents and larval behavior ${ }^{1}$. Although our understanding of ocean currents has greatly improved in the past decades allowing better reconstruction and prediction, a proper understanding of larval behavior has been more challenging due to a high degree of uncertainty and inter-specific variability ${ }^{2}$. In the past two decades, multiple studies demonstrated substantial swimming and orientation capabilities for larval fish of various species, which can affect their dispersal outcome ${ }^{3,4}$. However, most of these empirical studies focused on a single species at a single location, such that a generalized cross-species inference has rarely been attempted. This might be the reason for why larval behavior is not implemented in most biophysical larval dispersal models ${ }^{5}$. Here, we apply a cross-species meta-analysis focusing on the fundamental behavioral trait of larval directional swimming.

Recent studies have repeatedly demonstrated that fish larvae influence their dispersal by swimming directionally ${ }^{6}$. Directional precision is a central component in animal orientation $^{7,8}$ referring to the tendency of an individual to maintain a bearing and move along a straight path ${ }^{6,8}$. Directional precision can be achieved using internal stimuli or with reference to external cues, with only the latter representing orientation ${ }^{7}$. It is currently unclear which type is used by fish larvae. This is critical since larval dispersal is a key process governing demographic connectivity, gene flow and biogeography of marine populations ${ }^{9}$. Incorrect representation of larval orientation in biophysical models can lead to inaccurate estimations of larval transport and connectivity ${ }^{10}$. To test whether fish larvae use external cues for directional movement, we compare observed movement patterns to those expected under a strict use of internal cues. We find a robust support for the use of external cues by fish larvae, both at the individual and at the species level.

Significant directional precision has been demonstrated in fish larvae of more than 20 species $^{6}$, using two formats of field trials: (i) tracking larvae by scuba-divers (ScubaFollowing ${ }^{11}$ ) and (ii) video-recording of larvae inside the Drifting In Situ Chamber (DISC ${ }^{12}$ ). In both types of trials, the observed precision could be achieved by utilizing external or internal cues. Larvae that utilize internal cues may use their proprioceptive system, similar to a gyroscope $^{13}$, giving rise to a Correlated Random Walk (CRW) process whereby consecutive movement directions or 'bearings' are auto-correlated ${ }^{10}$. In contrast, "truly orienting" larvae 
use external cues such as the earth's magnetic field ${ }^{14}$, resulting in a Biased Random Walk $(B R W)$, whereby bearings correlate with a fixed external direction ${ }^{7,10}$. The distinction between the two is critical since external cues enable a more persistent directional movement over time ${ }^{7}$.

\section{Results}

The results of the Correlated Random Walk-von Mises (CRW-vm) analysis show that the observed mean directional precision $\left(\hat{R}_{\theta}\right)$ of 19 out of 21 tested species exceeded the mean expected under a strict use of internal cues $\left(\bar{R}_{\theta_{0}^{v m}}\right.$, Figure $1 \mathrm{~A}, \mathrm{~B}$, Table S1). However, in five species, confidence intervals overlap with $\bar{R}_{\theta_{0}^{v m}}$, including two species have means less than $\bar{R}_{\theta_{0}^{v m}}$ : Chaetodon aureofasciatus and Amblyglyphidodon curacao (Figure 1A,B, Table. S1). These two species do not display a clear use of external cues, likely because of their distinctive depth-dependent behavior (Supplementary Information, Section S1, Figure S1). In the three other species (Platax teira, Trachurus spp., and Epinephelus fuscoguttatus), the small number of trials (2-11) per species may explain the overlap (Table S1).

Fifteen species have enough trials $\left(N_{\text {trials }} \geq 20\right)$ for a within-species chi-square test (proportions: $0-50 \%, 50-75 \%$, and 75-100\%). Of these, thirteen species exhibit significant use of external cues for both $C R W$-vm and $C R W$-resampling $(C R W-r)$ analyses (chi-square test, $\mathrm{P}<0.0001$, Cohen's W>0.5; Table $\mathrm{S} 1$ ). The chi-square test results indicate a significant use of external cues as the distribution of effect sizes (Cohen's W) is significantly larger than 0.5 for $C R W$-vm and $C R W$-r (One sample t-test, $\mathrm{t}>4.9, \mathrm{P}<0.0001$ ).

$C R W$-vm and $C R W-r$ quantile analyses indicate a significant use of external cues for orientation because the $\hat{R}_{\theta}$ quantiles are significantly skewed towards higher values compared to the null ( $R_{\theta_{0}^{v m}}$ and $R_{\theta_{0}^{r}}$, Figure $1 \mathrm{C}$-D chi-square test, $\mathrm{P}<0.0001$, Cohen's $\mathrm{W}>0.5$ ). If larvae are using only internal cues, their density distribution should be centered around the means (Figure 1E, white circle). The high larval concentration on the top-right quarter of Figure 1E indicates that fish larvae use external cues.

\section{Discussion}

Previous work that quantitatively distinguished between internally and externally orienting animals, i.e., Correlated versus Biased Random Walk (CRW vs. BRW), have used: (i) step-length dynamics and their interactions with bearings ${ }^{15}$, (ii) displacement-related metrics for gauging directional precision, e.g., net squared displacement ${ }^{16,17}$, or (iii) net displacement $^{7,15}$. In contrast, we focus on bearings, constructing CRW-expected pattern using 
both theoretical and resampling techniques ${ }^{7,15,16,18,19}$. Specifically, we examine the movement dynamics based on the relationship between the $R_{\theta}$ and $R_{\Delta \theta}$. In our analyses, every larva is gauged against its own potential of autocorrelated directional precision, and can be readily compared against other larvae from either the same or different species (Figure 2).

CRW simulations assume a von Mises distribution of $\Delta \theta$, which is widely used to simulate $\mathrm{CRW}^{7,10,20-22}$. Yet, this distribution may not perfectly represent the real distribution of $\Delta \theta$. The strength of the sequence resampling $(C R W-r)$ analysis is that it makes no assumptions regarding the distribution of $\Delta \theta$, but instead reconstructs $\theta$ sequences by resampling the empirical $\Delta \theta^{7,16,23}$. Since these sequences are often short $\left(N_{o b s}=21\right)$, they may not properly represent the real underlying distribution. These two methods combined provide a complementary view and both lead to the conclusion of a robust use of external cues by orienting fish larvae.

Larval directional movement using external cues ${ }^{21,24-26}$ and autocorrelated swimming 21,22,27 were previously implemented in biophysical models of larval dispersal, largely demonstrating in both cases that these behaviors substantially affect dispersal, increase settlement success and alter connectivity patterns ${ }^{21,22,24-26}$. The mathematical methodology behind such an implementation was generally described in ${ }^{10}$, and is largely based on a sampling of swimming directions from a von Mises distribution centered around the direction of the cue source for orienting larvae, or around the swimming direction in the previous time step for autocorrelated movement ${ }^{21,22}$. In both cases, kappa, the von Mises distribution concentration parameter, governs the simulated precision of directional movement. Several studies used empirical species-specific data from in situ orientation trials to parametrize orientation behavior ${ }^{21,25}$. Yet, the combination and inter-dependence between external cues orientation and autocorrelated larval swimming direction were not implemented. The methodology presented here provides the basis for such implementation given the consideration of both orientation and autocorrelated directional swimming under a single framework.

Previous studies have demonstrated the need to consider larval orientation to understand the observed settlement success and connectivity ${ }^{28}$. It is therefore important to implement experimentally obtained orientation patterns into biophysical models, and the analytical approach presented here is key to increase the biological realism of larval dispersal models. Yet available orientation trials are of limited duration and longer trials are needed to 
study how orientation and its related cues change across time, space, and ontogeny. Future work should thus focus on isolating specific orientation cues, as well as studying cool-water, non-perciform taxa of commercial importance (e.g., Pleuronectiformes and Gadiformes) to promote a sustainable management of marine fish populations. Our meta-analysis demonstrates that fish larvae make extensive use of external cues for orientation, enabling them to find their way in the seemingly featureless, open ocean.

\section{Methods}

\section{General methodological approach}

To examine if larvae utilize external cues to generate directional movement, we develop two complementary analyses that compare the empirically observed directional precision with that expected under a strict use of internal cues. The empirically observed precision is quantified as the mean vector length $(R)$ of larval bearings $(\theta)$ (Figure $2 \mathrm{~A}$ ), herein $\hat{R}_{\theta}$. The angular differences between consecutive bearings, herein angle changes (Figure $2 \mathrm{~A} ; \Delta \theta_{t}=\theta_{t}-\theta_{t-1}$ ), is used to generate two null distributions of $R_{\theta}$ expected under a strict use of internal cues $\left(R_{\theta_{0}}\right)$. The first is theoretical and is based on a von Mises distribution of simulated $\Delta \theta$, herein $C R W$ $v m$ (Figure 2B,C); the second is empirical, and is based on resampling the $\Delta \theta$ within each trial, herein $C R W$-r (Figure 2D,E). These two analyses are complementary because the first can generate unlimited number of trajectories but is based on a theoretical distribution rather than on observations, whereas the second is based on observations which are limited in sample size.

\section{Quantitative analyses}

We compute the quantiles in which the observed precision $\left(\hat{R}_{\theta}\right)$ of each trial falls within the null distributions $\left(R_{\theta_{0}^{v m}}\right.$ and $\left.R_{\theta_{0}^{r}}\right)$, and compare these quantile distributions with the null quantile distributions using a chi-square test, gauging the observed directional precision $\hat{R}_{\theta}$ against the potential of autocorrelated precision $\left(R_{\theta_{0}}\right)$. For individuals using external cues for orientation, the empirical $\hat{R}_{\theta}$ is expected to exceed the autocorrelated pattern, $R_{\theta_{0}}$, whereas for a strictly internal-cues-utilizing individual, $\hat{R}_{\theta}$ is expected to be closer to $\bar{R}_{\theta_{0}}{ }^{7}$ (Figure 2C). $\hat{R}_{\theta}$ values less than $R_{\theta_{0}}$ may result from a one-sided bias (left or right) or from a multimodal distribution of $\theta$, representing the utilization of internal and external cues, respectively (Figure 2C, Supplementary Information S1). We apply our methods to a database of 782 in situ orientation trials gathered on larvae of 21 species from eight families of perciform fishes at 
various tropical and warm-water locations (East Asia, Australia, Mediterranean and Red Sea; Table S1).

The methodology used for these in situ studies can be divided into two main categories. First, with direct observations through Scuba-Following, where a larva is released in the pelagic environment and tracked by scuba divers for 10 min during which swimming direction is recorded every $30 \mathrm{~s}$, resulting in 21 observations $\left(N_{o b s}=21\right)$; for the full protocol, see ${ }^{11,29}$. Second, with observations using the Drifting In Situ Chamber $\left(D I S C^{30}\right)$. For each DISC trial, a larva is placed into a circular chamber, and its position is recorded for $15-20$ min with a camera fixed circa $50 \mathrm{~cm}$ below the chamber. The first 3-5 minutes of each DISC trial are considered as acclimation time and are excluded from the analysis, whereas the residual 10-15 min are the actual observations used for the analysis. The number of observations per deployment $\left(N_{o b s}=90,180\right.$ or 300 ; see Table S1) varies with the recording frequency of larval positions, ranging from 2 to 10 seconds (Table S1). It is noteworthy that these two methods have been tested together and have demonstrated high consistency in larval orientation results ${ }^{31,32}$.

Each orientation trial includes a sequence of larval swimming directions, termed bearings $(\theta)$ (Figure 2A). For the DISC trials, $\theta$ are the cardinal directions of larval positions within the DISC's chamber ${ }^{33}$. The angular differences between $\theta$ of consecutive time steps $(t)$ are defined as $\Delta \theta\left(\Delta \theta_{t}=\theta_{t}-\theta_{t-1}\right)$, such that for every $\theta$ sequence of a given length $(N)$, there is a respective $\Delta \theta$ sequence of length $N-1$ (Figure 2A). Directional precision with respect to external and internal cues is computed as the mean vector length of bearings $(R \theta)$ and of angle changes $\left(R_{\Delta \theta}\right)$, respectively ${ }^{30}$. Values of mean vector length $(R)$ range from 0 to 1 , with 0 indicating a uniform distribution of angles and 1 indicating that all angles are the same.

We used two quantitative approaches to examine if larvae utilize external cues for orientation: the "Correlated Random Walk-von Mises" (CRW-vm) analysis and the "CRW resampling" (CRW-r) analysis. Both analyses are based on the principle that trajectories of animals that strictly use internal cues for directional movement are characterized by a $C R W$ pattern. Hence, their capacity for directional movement is exclusively dependent on the distribution of their angle changes $(\Delta \theta)^{34}$. In contrast, for an external-cues orienting animal, for which movement directions are correlated with an external fixed direction, the directional precision of the observed bearings, $\hat{R}_{\theta}$, is expected to exceed the directional precision of bearings following a $C R W, R_{\theta_{0}}{ }^{7}$. Both analyses compare $\hat{R}_{\theta}$ against the expected $R_{\theta_{0}}$, but 
the first analysis computes $R_{\theta_{0}^{v m}}$ using a theoretical von Mises distribution of $\Delta \theta$, and the second computes $R_{\theta_{0}^{r}}$ by producing 100 new $\theta$ sequences per individual trial (larva) by multiple resampling-without-replacement of the $\Delta \theta$. A key principle for both analyses stems from the fact that the mean vector length of bearings $\left(R_{\theta}\right)$ is inherently dependent on the mean vector length of angle changes $\left(R_{\Delta \theta}\right)$. In other words, an animal with a high capacity for internal-cue directional movement, i.e., a narrow distribution of $\Delta \theta$, is likely to yield a high $R_{\theta}$ even if it makes absolutely no use of external cues for orientation. Hence, in both analyses, to determine a use of external cues, $\hat{R}_{\theta}$ is gauged against a distribution of $R_{\theta_{0}}$, given its respective precision of angle changes $\hat{R}_{\Delta \theta}$. The open-source software $R^{35}$ with the package "circular" 36 is used for all analyses in this study.

Correlated Random Walk-von Mises (CRW-vm)

In this analysis, we first generate the directional precision $(R)$, expected under a strict use of internal cues using the theoretical von Mises distribution $\left(R_{\theta_{0}^{v m}}\right)$. The $C R W$ bearings sequences $\left(\theta_{0}^{v m}\right)$ are generated by choosing a random initial bearing, followed by a series of $N_{\text {obs }}-1$ angle changes $\left(\Delta \theta_{0}^{v m}\right)$ in bearing direction; drawn at random (with replacement) from a von Mises distribution $\left(N_{r e p}=1000\right)$. The length of $\theta_{0}^{v m}$ sequence is according to the number of observations in our four types of experimental trials: $N_{o b s}=21$ for the Scuba-following, and 90, 180 and 300 for the DISC (Table S1). The directional precision of the von Mises distribution is dependent on the concentration parameter, kappa. Kappa values ranging from 0 to 399 are applied at 1-unit increments to cover the entire range of directional precision from completely random (kappa=0), to highly directional (kappa=399). Next, the directional precision of the bearings $\left(R_{\theta}\right)$ and the angle changes $\left(R_{\Delta \theta}\right)$ are computed for each simulated sequence of $\theta$ (Figure $2 \mathrm{~A}-\mathrm{C}$ ).

These respective pairs of values $\left(R_{\Delta \theta}, R_{\theta}\right)$ provide the basis for generating the expected relationship between $R_{\theta_{0}^{v m}}$ and $R_{\Delta \theta_{0}^{v m}}$. Then, for any given kappa value, the following quantiles are computed: $5^{\text {th }}, 10^{\text {th }}, 20^{\text {th }}, \ldots, 90^{\text {th }}$, and $95^{\text {th }}$ (grey vertical distributions in Figure $2 \mathrm{C})$. Next, smooth spline functions are fitted through all respective quantiles, generating the $R_{\theta_{0}^{v m}}$ quantiles contours, which represents the null expectation under a strict use of internal cues. This expected $\left(R_{\Delta \theta}, R \theta\right)$ correspondence creates a phase diagram (Figure $2 \mathrm{C}$ ), based on which the observed $\theta$ patterns are gauged. The procedure is repeated four times to match the 
among-study differences in the number of $\theta$ observations per trial (i.e., $N_{o b s}=21,90,180$, and 300; see Table S1).

To examine if the observed larval movement patterns are different from those expected under a pure use of internal cues $(C R W-v m)$, we compute $R_{\Delta \theta}$ and $R_{\theta}$ for the trial sequences of each individual larva $\left(\hat{R}_{\Delta \theta}\right.$ and $\left.\hat{R}_{\theta}\right)$. We then place these values in the phase diagram and examine their positions with respect to $R_{\theta_{0}^{v m}}$ (Figure $2 \mathrm{C}$ ). Larvae with $\hat{R}_{\theta}$ substantially higher than $\bar{R}_{\theta_{0}^{v m}}$, are considered to have a higher tendency for using external cues for orientation (Figure $2 \mathrm{~B}, \mathrm{C})^{7,15}$. Larvae with $\hat{R}_{\theta}$ values substantially below $\bar{R}_{\theta_{0}^{v m}}$ indicate a multimodal $\theta$ distribution or a one-sided drift (right or left). A larva is considered directional if the bearings sequence $(\hat{\theta})$ is significantly different from a uniform distribution based on the Rayleigh's test $(p<0.05)$. Non-directional larvae are characterized by low $\hat{R}_{\Delta \theta}$ and $\hat{R}_{\theta}$, and thus will be situated at the bottom left area in the phase diagram (Figure $2 \mathrm{C}$ ).

The quantile $(Q)$ of each trial is then computed based on the location of $\left(\hat{R}_{\Delta \theta}, \hat{R}_{\theta}\right)$ within the null quantiles contours in the phase diagram (Figure 2C), using a 2-D interpolation such that $X=R_{\Delta \theta}, Y=R \theta$, and $Z=Q$ ("Akima" R package ${ }^{37}$; Figure 1B). 2-D interpolation is used once more to overlay the $\left(\hat{R}_{\Delta \theta}, \hat{R}_{\theta}\right)$ of two species with a different number of observations ( $N_{o b s}=300$ : Premnas biaculeatus, $N_{o b s}=90$ Chromis atripectoralis) on the DISC's phase diagram $\left(N_{o b s}=180\right)$, which represents most DISC trials.

\section{Correlated Random Walk- resampling (CRW-r)}

In this analysis, we generate $R_{\theta_{0}}$ expected under a strict use of internal cues using resampling of the angle changes $(\Delta \theta)$ per trial sequence (i.e., $R_{\theta_{0}^{r}}$ ). Specifically, for every trial sequence, $R_{\theta_{0}^{r}}$ is computed by generating $100 \theta$ sequences from 100 resampled $\Delta \theta$ sequences ( $N_{r e p}=100$, without replacement) from the empirical $\Delta \theta$ (Figure 2D). The $R_{\theta_{0}^{r}}$ sequence length is equal to the number of observations in each trial $\left(N_{o b s}\right)$. Next, $R_{\theta}$ for each of the resampled sequences $\left(R_{\theta_{0}^{r}}\right)$ and the quantile in which $\hat{R}_{\theta}$ falls within $R_{\theta_{0}^{r}}$, are computed (Figure 2E). The quantile represents the proportion of $R_{\theta_{0}^{r}}$ which is smaller than $\hat{R}_{\theta}$ for each trial. 
To test if $\hat{R}_{\theta}$ were significantly higher than what is expected under the null, we used chisquare tests to compare the $\hat{R}_{\theta}$ quantile distributions (trial counts) with the null ( $R_{\theta_{0}^{v m}}$ and $\left.R_{\theta_{0}^{r}}\right)$ quantile distributions. For applying chi-square tests on all larvae pooled together, we used the following quantile partitioning: $0-50 \%, 50-70 \%, 70-90 \%$, and $90-100 \%$. For applying chi-square tests on larvae pooled by species, we used the following quantile partitioning: $0-50 \%, 50-75 \%, 75-100 \%$. The reason for the differences is the "minimal samples number" limitation of the chi-square goodness-of-fit test, which is a minimum of 5 samples per expected bin $^{38}$. This limitation allows a minimum of 20 samples in the species chi-square test, and 50 samples in the chi-square test for all larvae pooled together.

To test weather $\hat{R}_{\theta}$ are significantly higher than what is expected under the null across species, we computed the effect size (Cohen's W) of each chi-square test per species, and examined if the effect sizes distribution is significantly higher than 0.5 using a one-sample ttest. Effect size of Cohen's $W \geq 0.5$ represents a strong effect size for the chi-square goodnessof-fit test ${ }^{39}$.

To examine the correspondence between the $\hat{R}_{\theta}$ quantiles of the two methods: $C R W$ $v m$ and $C R W-r$, the quantiles $(Q)$ data were binned at increments of $5 \%$ for the two analyses, creating a 20x20 cell matrix $(M)$. Then, the matrix was filled based on the $\hat{R}_{\theta}$ quantiles of the larvae, such that for example, a given larva with $Q_{C R W-v m}=99 \%$ and $Q_{C R W-r}=96 \%$, will be counted in $M_{20,20}$. Whereas, a given larva with $Q_{C R W-v m}=52 \%$ and $Q_{C R W-r}=46 \%$, will be counted in $M_{11,10}$. Based on this matrix, the corresponding heatmap contoured plot in Figure $1 \mathrm{E}$ was produced, using $\mathrm{R}$ package “plot3D”40.

If the two methods of analysis ( $C R W-v m$ and $C R W-r)$ provide significant test results for a given species, this can be regarded as strong evidence for the use of external cues for orientation. If both methods fail to reject the use of internal stimuli, it seems likely that external cues are not used for directional swimming. However, if the two methods provide differing test results, no definitive conclusion about how directional precision is maintained can be reached. 


\section{Author contributions}

IB, CBP, MK: conceptualization. IB, RF, JOI, MK, UES, JML, CBP: investigation. IB: formal analysis, visualization. RF, JOI, MK, UES, JML, CBP: validation. IB; initial draft. IB, RF, JOI, MK, UES, JML, CBP: writing, review and editing.

\section{Declaration of interests}

The authors declare no competing interests.

\section{Acknowledgments}

We would like to thank everyone who have contributed to these valuable datasets over the years. I.B. was funded by the National Academy of Science, Engineering and Medicine - Gulf research Program NASEM-GRP award number 2000007703 to Steven Murawski and C.B.P; R.F. and J.O.I were funded by the Paris Lab of the Rosenstiel School of Marine and Atmospheric Sciences and a grant from the Partner University Fund (PUF); M.K. was funded by the Binational Science Foundation BSF Grant number 2008144; J.M.L. and U.S. were funded by the Australia Research Council (ARC) Discovery Grant DP110100695. The Study was made possible through a National Science Foundation Award NSF-OCE 1459156 to C.B.P.

\section{References}

1. Cowen, R. K. \& Sponaugle, S. Larval dispersal and marine population connectivity. Mar. Sci. 1, 443-466 (2009).

2. Barth, P. et al. From the ocean to a reef habitat: How do the larvae of coral reef fishes find their way home? A state of art on the latest advances. Vie Milieu 65, 91-100 (2015).

3. Leis, J. M. Are larvae of demersal fishes plankton or nekton? Adv. Mar. Biol. 51, 57-141 (2006).

4. Leis, J. M., Siebeck, U. \& Dixson, D. L. How Nemo finds home: the neuroecology of dispersal and of population connectivity in larvae of marine fishes. Integr. Comp. Biol. 51, 826-843 (2011).

5. Leis, J. M. Perspectives on larval behaviour in biophysical modelling of larval dispersal in marine, demersal fishes. in Oceans vol. 2 1-25 (Multidisciplinary Digital Publishing Institute, 2021).

6. Leis, J. M. Are larvae of demersal fishes plankton or nekton? Adv. Mar. Biol. 51, 57-141 (2006).

7. Nams, V. O. Detecting oriented movement of animals. Anim. Behav. 5, 1197-1203 (2006).

8. Dacke, M., Baird, E., Byrne, M., Scholtz, C. H. \& Warrant, E. J. Dung beetles use the Milky Way for orientation. Curr. Biol. 23, 298-300 (2013).

9. Cowen, R. K., Paris, C. B. \& Srinivasan, A. Scaling of connectivity in marine populations. Science (80). 311, 522-527 (2006).

10. Codling, E. A., Hill, N. A., Pitchford, J. W. \& Simpson, S. D. Random walk models for the movement and recruitment of reef fish larvae. Mar. Ecol. Prog. Ser. 279, 215-224 (2004).

11. Leis, J. M., Sweatman, H. P. A. \& Reader, S. E. What the pelagic stages of coral reef fishes 
are doing out in blue water: daytime field observations of larval behavioural capabilities. Mar. Freshw. Res. 47, 401-411 (1996).

12. Paris, C. B. et al. Reef odor: a wake up call for navigation in reef fish larvae. PLoS One $\mathbf{8}$, e72808 (2013).

13. Williams IV, R., Neubarth, N. \& Hale, M. E. The function of fin rays as proprioceptive sensors in fish. Nat. Commun. 4, 1729 (2013).

14. Bottesch, M. et al. A magnetic compass that might help coral reef fish larvae return to their natal reef. Curr. Biol. 26, R1266-R1267 (2016).

15. Benhamou, S. Detecting an orientation component in animal paths when the preferred direction is individual-dependent. Ecology 87, 518-528 (2006).

16. Kareiva, P. M. \& Shigesada, N. Analyzing insect movement as a correlated random walk. Oecologia 56, 234-238 (1983).

17. Nolet, B. A. \& Mooij, W. M. Search paths of swans foraging on spatially autocorrelated tubers. J. Anim. Ecol. 71, 451-462 (2002).

18. Bailey, J. D., Wallis, J. \& Codling, E. A. Navigational efficiency in a biased and correlated random walk model of individual animal movement. Ecology 99, 217-223 (2018).

19. Huebert, K. B. \& Sponaugle, S. Observed and simulated swimming trajectories of late-stage coral reef fish larvae off the Florida Keys. Aquat. Biol. 7, 207-216 (2009).

20. Marsh, L. M. \& Jones, R. E. The form and consequences of random walk movement models. J. Theor. Biol. 133, 113-131 (1988).

21. Berenshtein, I. et al. Biophysical simulations support schooling behavior of fish larvae throughout ontogeny. Front. Mar. Sci. 5, 254 (2018).

22. Berenshtein, I. et al. Auto-correlated directional swimming can enhance settlement success and connectivity in fish larvae. J. Theor. Biol. 439, 76-85 (2018).

23. Papastamatiou, Y. P. et al. Scales of orientation, directed walks and movement path structure in sharks. J. Anim. Ecol. 80, 864-874 (2011).

24. Staaterman, E., Paris, C. B. \& Helgers, J. Orientation behavior in fish larvae: A missing piece to Hjort's critical period hypothesis. J. Theor. Biol. 304, 188-196 (2012).

25. Faillettaz, R., Paris, C. B. \& Irisson, J.-O. Larval fish swimming behavior alters dispersal patterns from marine protected areas in the north-western Mediterranean sea. Front. Mar. Sci. 5, 97 (2018).

26. Wolanski, E. \& Kingsford, M. J. Oceanographic and behavioural assumptions in models of the fate of coral and coral reef fish larvae. J. R. Soc. Interface 11, 20140209 (2014).

27. Porch, C. E. A numerical study of larval fish retention along the southeast Florida coast. Ecol. Modell. 109, 35-59 (1998).

28. Bode, M. et al. Successful validation of a larval dispersal model using genetic parentage data. PLoS Biol. 17, e3000380 (2019).

29. Leis, J. M. et al. In situ orientation of fish larvae can vary among regions. Mar. Ecol. Prog. Ser. 537, 191-203 (2015).

30. Paris, C. B., Guigand, C. M., Irisson, J., Fisher, R. \& D’Alessandro, E. Orientation with no 
frame of reference (OWNFOR): a novel system to observe and quantify orientation in reef fish larvae. in Implications for Marine Protection Area Management, NOAA National Marine Sanctuary Program 52-62 (2008). doi:10.1046/j.1467-2960.2001.00053.x.

31. Leis, J. M., Paris, C. B., Irisson, J.-O., Yerman, M. N. \& Siebeck, U. E. Orientation of fish larvae in situ is consistent among locations, years and methods, but varies with time of day. Mar. Ecol. Prog. Ser. 505, 193-208 (2014).

32. Irisson, J.-O., Paris, C. B., Leis, J. M. \& Yerman, M. N. With a little help from my friends: group orientation by larvae of a coral reef fish. PLoS One 10, e0144060 (2015).

33. Irisson, J. O., Guigand, C. \& Paris, C. B. Detection and quantification of marine larvae orientation in the pelagic environment. Limnol Ocean. Methods 7, 664-672 (2009).

34. Cheung, A., Zhang, S., Stricker, C. \& Srinivasan, M. V. Animal navigation: the difficulty of moving in a straight line. Biol. Cybern. 97, 47-61 (2007).

35. R Foundation. The R Project for statistical computing. (2017).

36. Agostinelli, C. \& Lund, U. R package 'circular': Circular Statistics (version 0.4-3). URL https://r-forge.r-project.org/projects/circular (2011).

37. Akima, H., Gebhardt, A., Petzold, T. \& Maechler, M. Package 'akima'. (2016).

38. McDonald, J. H. Handbook of biological statistics. vol. 2 (sparky house publishing Baltimore, MD, 2009).

39. Cohen, J. A power primer. Psychol. Bull. 112, 155 (1992).

40. Soetaert, K. plot3D: Tools for plotting 3-D and 2-D data. $R$ Packag. version 10-12 (2014). 
(A)

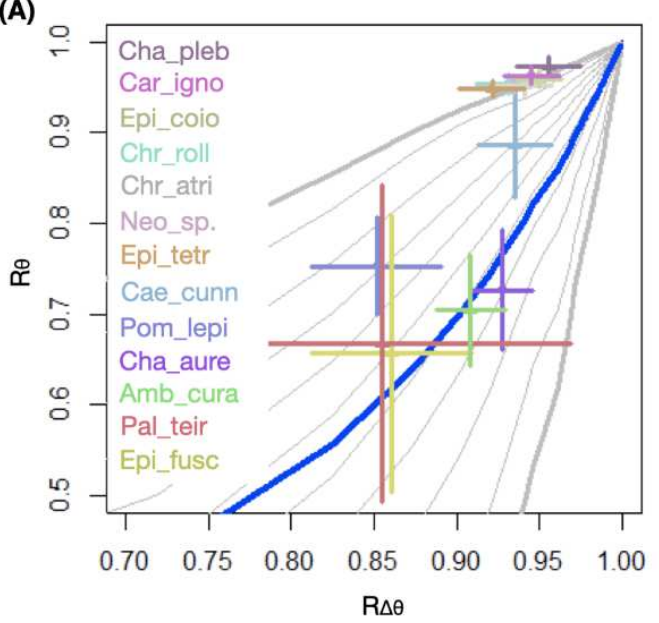

(C)

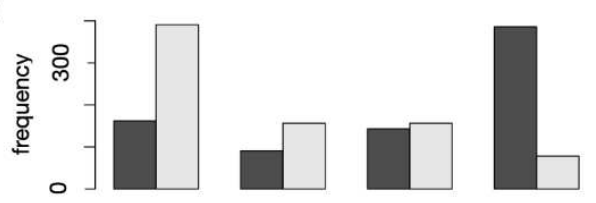

(D)

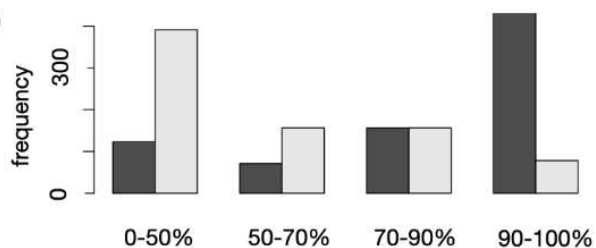

(B)

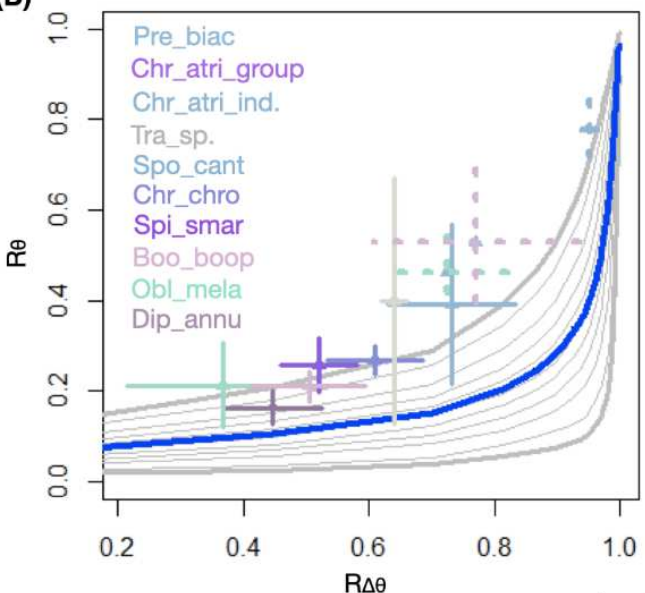

(E)

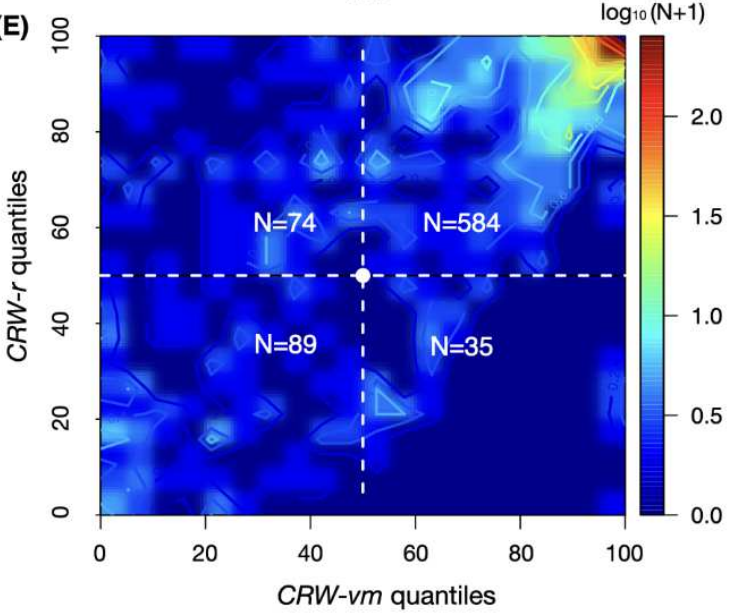

Figure 1. Results of two analytical methods applied for testing the use of external cues for orientation by fish larvae: Correlated Random Walk-von Mises $(C R W-v m)$, Correlated Random Walk resampling $(\boldsymbol{C R} W-r) . C R W$-vm analysis at the species level based on the diagram in (Figure 2C) for the scuba-following trials (A), and for the DISC trials (B) with various number of samples-per-trial: $N_{o b s}=21$ (A), 300, 90, and 180 (B). Crosses represent means $\pm 95 \% \mathrm{CI}$ of the observed $\left(R_{\Delta \theta}, R_{\theta}\right)$ pooled by species. Crosses with dashed lines in (B) represent species with $N_{o b s}$ different than 90 , see Table S1 for more details. Species names are ordered top-down according to their $R_{\theta}$ means and correspond in color to their respective crosses in (A, B). Species names abbreviations are: Amblyglyphidodon curacao (Amb_cura), Boops boops (Boo_boop), Caesio cuning (Cae_cuni), Caranx ignobilis (Car_igno), Chaetodon aureofasciatus (Cha_aure), Chaetodon plebius (Cha_pleb), Chromis atripectoralis (Chr_atri), Chromis chromis (Chr_chro), Chrysiptera rollandi (Chr_roll),Diplodus annularis (Dip_annu), Epinephelus coioides (Epi_coio), Epinephelus fuscoguttatus (Epi_fusc), Eleutheronema tetradactylum (Ele_tetr), Neopomacetrus cyanomos (Neo_cyan), Oblada melanura (Obl_mela), Platax teira (Pla_teir), Pomacentrus lepidogenys (Pom_lepi), Premnas biaculeatus (Pre_biac), Spicara smaris (Spi_smar), Spondyliosoma cantharus (Spo_cant), Trachurus spp. (Tra_spp.). Chi-square goodness of fit plots comparing the $\hat{R}_{\theta}$ quantiles distributions (black bars) to the null quantile distributions (grey bars) for $C R W-v m$ (C) and $C R W-r$ (D). (E) A heatmap of trial counts binned at 5\% increments according to their $\hat{R}_{\theta}$ quantiles in the $C R W-v m$ and $C R W-r$ analyses. 


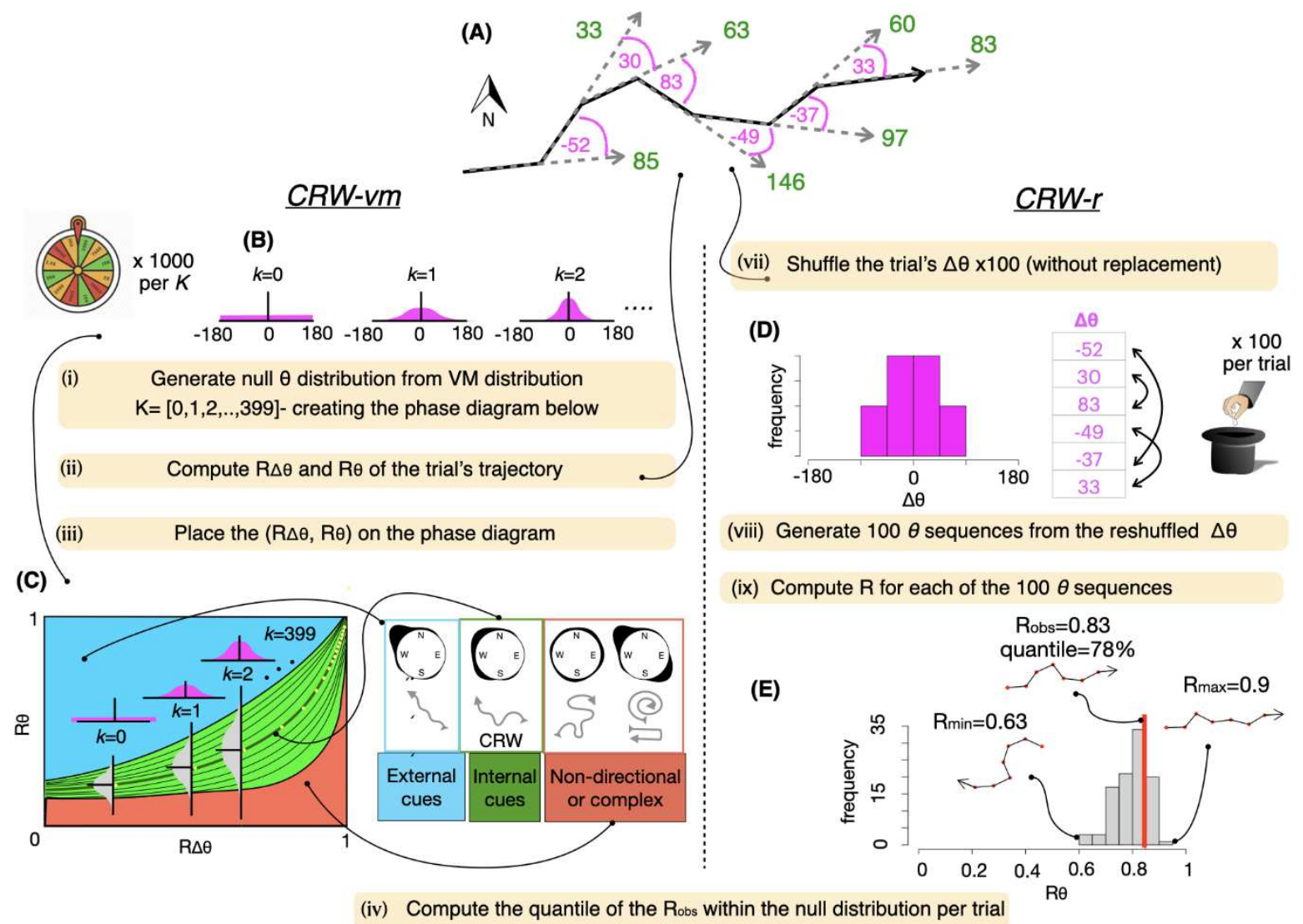

(v) Apply chi-square goodness of fit tests and compute their effect sizes (Cohen's W) for all larvae and by species

(vi) Apply one sample t-test on the Cohen's W effect size to test the overall effect

Figure 2. Two analytical methods applied for testing the use of external cues for orientation by fish larvae: Correlated Random Walk-von Mises (CRW-vm; left section), Sequence Resampling ( $\boldsymbol{C R} \boldsymbol{W}$-r; right section). (A) Each trial includes a bearings sequence ( $\theta$; green), from which the angle changes sequence ( $\Delta \theta$; magenta) is computed. For visual clarity, (A) includes only seven bearings $\left(N_{o b s}=7\right)$, while in the actual trials $N_{o b s}$ is larger. (i) To generate the $C R W$-vm null distribution, we sample (with replacement) from a von Mises distribution, generating for each $K$ value $(K: 1,2,3 \ldots, 399), 1000 \Delta \theta$ sequences and corresponding $\theta$ sequences at the same length as $N_{o b s}$ per trial. (ii) For each of these simulated sequences, mean vector lengths $(R)$ of $\Delta \theta$ and $\theta$ are computed $(R \Delta \theta, R \theta)$, such that a distribution of $R \theta$ (grey vertical distributions in (C)) is generated for each $K$ (yellow dots). The $5^{\text {th }}, 10^{\text {th }}, 20^{\text {th }}, \ldots ., 90^{\text {th }}$, and $95^{\text {th }}$ quantiles of each of these $R \theta$ distributions is computed, and contours connect these quantiles across the different $R_{\theta}$ distributions (black contours in $(C)$ ), with the mean represented in dark green line. Green area in $(C)$ represents directional precision $\left(R_{\theta}\right)$ expected under pure $C R W\left(R_{\theta_{0}^{v m}}\right)$, such that samples with $R_{\theta}$ closer to the mean indicate a tendency for internal cues, whereas samples with high $R_{\theta}$ compared to $R_{\theta_{0}^{v m}}$ (closer to the blue area), represent a tendency for external cues. The orange area below $R_{\theta_{0}^{v m}}$, represents: non-directional larvae, one-sided bias (i.e., right/left) or multimodal distribution of bearings, respectively (for more details see Supplementary section $\mathrm{S} 1$ ). (iii) $\left(R_{\Delta \theta}, R_{\theta}\right)$ is computed per experimental trial, and plotted on the phase diagram both individually and by species. (iv) Quantiles of the observed $R_{\theta}\left(\hat{R}_{\theta}\right)$ within the $R_{\theta_{0}^{v m}}$ are computed using a 2-D 
interpolation. (v) Chi-square tests are applied to compare the $\hat{R}_{\theta}$ and the $R_{\theta_{0}^{v m}}$ distributions; and the effect size of the chi-square test (Cohen's W) is computed. (vi) A one sample t-test is applied to examine the significance of the effect size (Cohen's W > 0.5) across all species. Correlated Random Walk- resampling ( $\boldsymbol{C R} W$-r; right section). For each trial, $\Delta \theta$ is computed (A), as shown in the histogram (D) of the example sequence in (A). Then, $\Delta \theta$ sequence is sampled without replacement 100 times (vii), and $100 \theta$ sequences are generated (viii). Next, (E) $R$ is computed for each of these $100 \theta$ sequences $\left(R_{\theta_{0}^{r}}\right)$ (ix), and the quantile of the $\hat{R}_{\theta}$ within the $R_{\theta_{0}^{r}}$ distribution is calculated (iv), in this example quantile $=78 \%$. Then, stages (iv-vi) are applied the same as for the $C R W$-vm method, ultimately computing the strength of the differences between the $\hat{R}_{\theta}$ and $R_{\theta_{0}^{r}}$ quantile distributions. 


\section{Figures}

(A)

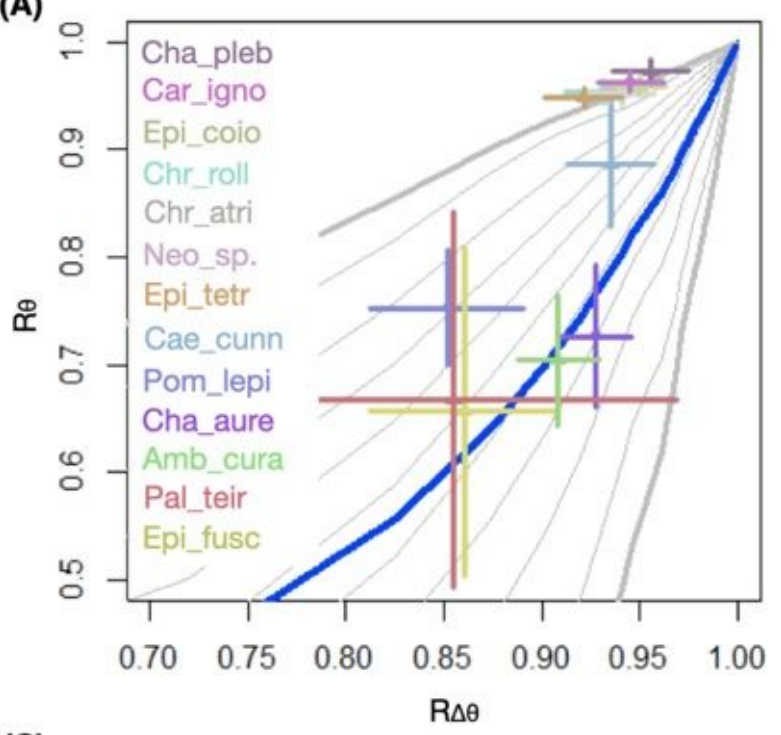

(C)

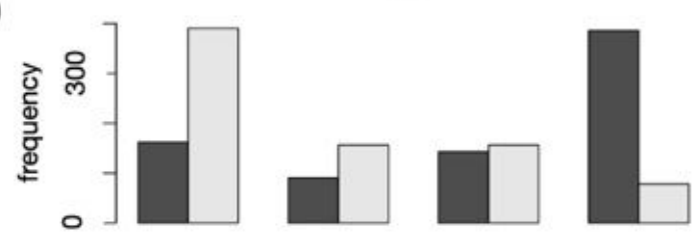

(D)

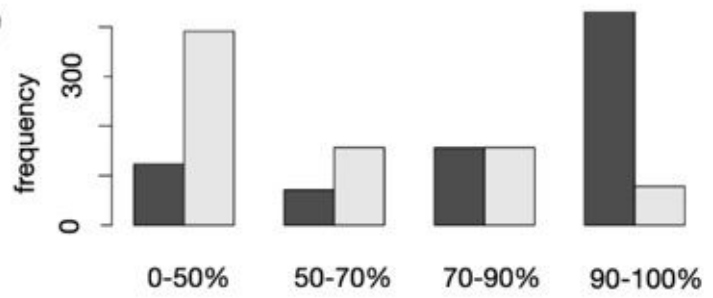

(B)
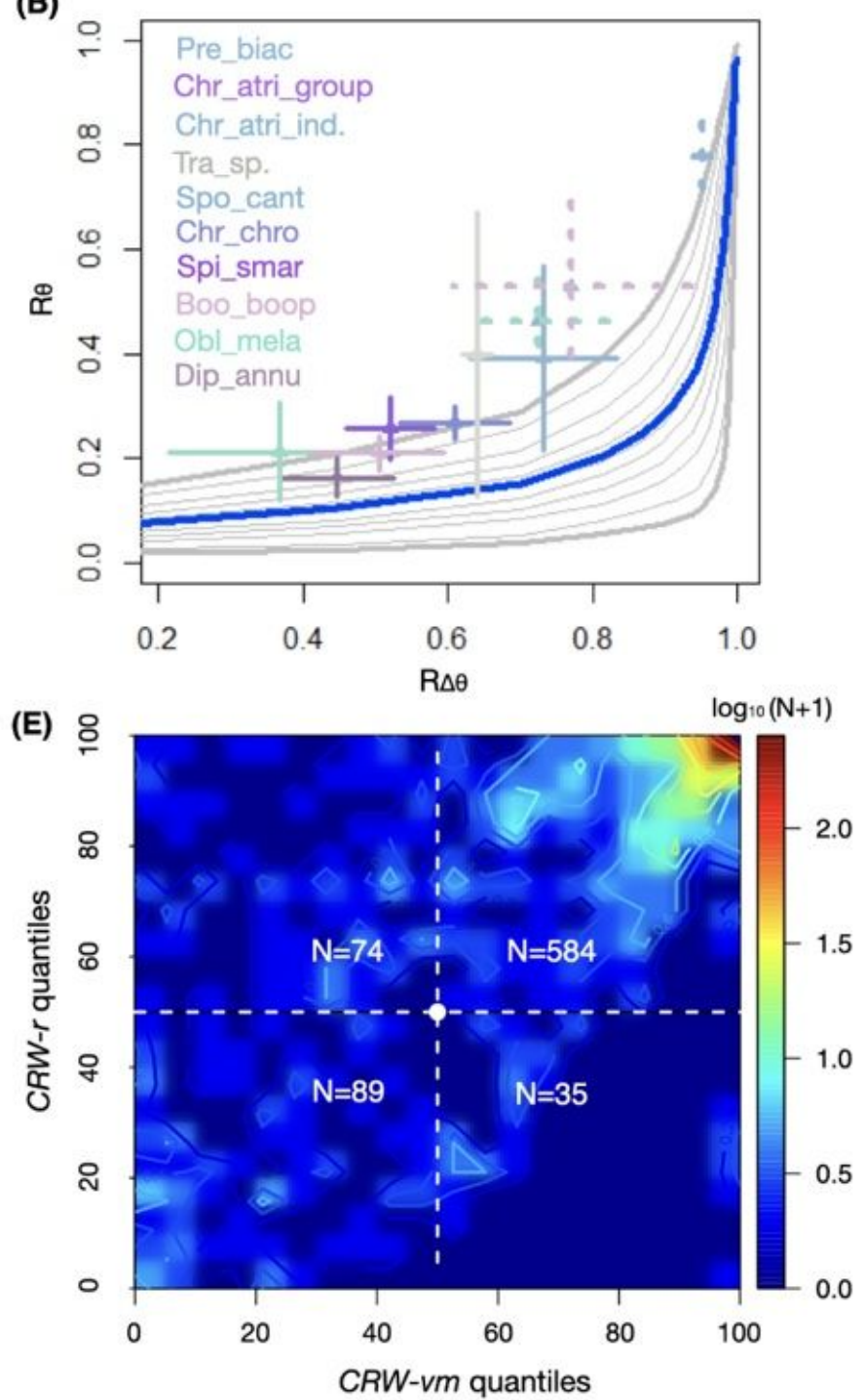

\section{Figure 1}

Results of two analytical methods applied for testing the use of external cues for orientation by fish larvae: Correlated Random Walk-von Mises (CRW-vm), Correlated Random Walk resampling (CRW-r). CRW-vm analysis at the species level based on the diagram in (Figure 2C) for the scuba-following trials (A), and for the DISC trials (B) with various number of samples-per-trial: Nobs=21 (A), 300, 90, and 180 (B). Crosses represent means $\pm 95 \% \mathrm{Cl}$ of the observed ( $\mathrm{R} \Delta \theta, \mathrm{R} \theta)$ pooled by species. Crosses with dashed lines in (B) represent species with Nobs different than 90, see Table S1 for more details. Species names are ordered top-down according to their $\mathrm{R} \theta$ means and correspond in color to their respective crosses in (A, B). Species names abbreviations are: Amblyglyphidodon curacao (Amb_cura), Boops boops (Boo_boop), Caesio cuning (Cae_cuni), Caranx ignobilis (Car_igno), Chaetodon aureofasciatus (Cha_aure), Chaetodon plebius (Cha_pleb), Chromis atripectoralis (Chr_atri), Chromis chromis (Chr_chro), Chrysiptera rollandi (Chr_roll),Diplodus annularis (Dip_annu), Epinephelus coioides (Epi_coio), 
Epinephelus fuscoguttatus (Epi_fusc), Eleutheronema tetradactylum (Ele_tetr), Neopomacetrus cyanomos (Neo_cyan), Oblada melanura (Obl_mela), Platax teira (Pla_teir), Pomacentrus lepidogenys (Pom_lepi), Premnas biaculeatus (Pre_biac), Spicara smaris (Spi_smar), Spondyliosoma cantharus (Spo_cant), Trachurus spp. (Tra_spp.). Chi-square goodness of fit plots comparing the quantiles distributions (black bars) to the null quantile distributions (grey bars) for CRW-vm (C) and CRW-r (D). (E) A heatmap of trial counts binned at $5 \%$ increments according to their quantiles in the CRW-vm and CRW-r analyses.

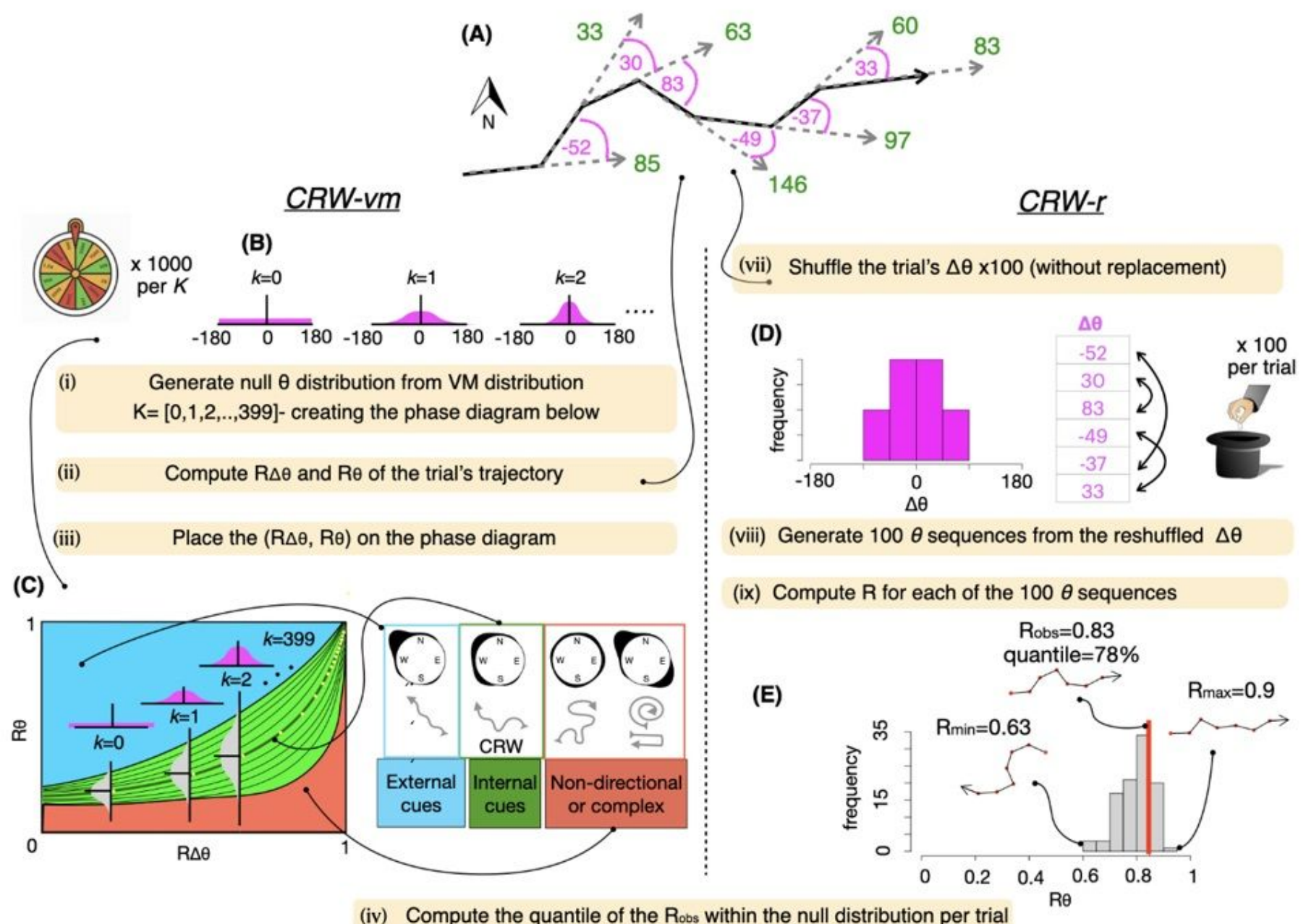

(v) Apply chi-square goodness of fit tests and compute their effect sizes (Cohen's W) for all larvae and by species

(vi) Apply one sample t-test on the Cohen's W effect size to test the overall effect

\section{Figure 2}

Two analytical methods applied for testing the use of external cues for orientation by fish larvae: Correlated Random Walk-von Mises (CRW-vm; left section), Sequence Resampling (CRW-r; right section). (A) Each trial includes a bearings sequence $(\theta$; green), from which the angle changes sequence $(\Delta \theta$; magenta) is computed. For visual clarity, $(A)$ includes only seven bearings (Nobs=7), while in the actual trials Nobs is larger. (i) To generate the CRW-vm null distribution, we sample (with replacement) from a von Mises distribution, generating for each $\mathrm{K}$ value (K:1,2,3..., 399), $1000 \Delta \theta$ sequences and corresponding $\theta$ sequences at the same length as Nobs per trial. (ii) For each of these simulated 
sequences, mean vector lengths ( $R$ ) of $\Delta \theta$ and $\theta$ are computed $(R \Delta \theta, R \theta)$, such that a distribution of $R \theta$ (grey vertical distributions in (C)) is generated for each $\mathrm{K}$ (yellow dots). The 5th, 10th, 20th, ...., 90th, and 95th quantiles of each of these R $\theta$ distributions is computed, and contours connect these quantiles across the different $\mathrm{R} \theta$ distributions (black contours in (C)), with the mean represented in dark green line. Green area in (C) represents directional precision (R $\theta)$ expected under pure CRW ( ), such that samples with $\mathrm{R} \theta$ closer to the mean indicate a tendency for internal cues, whereas samples with high $\mathrm{R} \theta$ compared to (closer to the blue area), represent a tendency for external cues. The orange area below , represents: non-directional larvae, one-sided bias (i.e., right/left) or multimodal distribution of bearings, respectively (for more details see Supplementary section $\mathrm{S} 1$ ). (iii) (R $\Delta \theta, \mathrm{R} \theta)$ is computed per experimental trial, and plotted on the phase diagram both individually and by species. (iv) Quantiles of the observed R $\theta$ () within the are computed using a 2-D interpolation. (v) Chi-square tests are applied to compare the and the distributions; and the effect size of the chi-square test (Cohen's W) is computed. (vi) A one sample t-test is applied to examine the significance of the effect size (Cohen's $W>0.5$ ) across all species. Correlated Random Walk- resampling (CRW-r; right section). For each trial, $\Delta \theta$ is computed (A), as shown in the histogram (D) of the example sequence in (A). Then, $\Delta \theta$ sequence is sampled without replacement 100 times (vii), and $100 \theta$ sequences are generated (viii). Next, (E) R is computed for each of these $100 \theta$ sequences () (ix), and the quantile of the within the distribution is calculated (iv), in this example quantile $=78 \%$. Then, stages (iv-vi) are applied the same as for the CRW-vm method, ultimately computing the strength of the differences between the and quantile distributions.

\section{Supplementary Files}

This is a list of supplementary files associated with this preprint. Click to download.

- SupplemetaryinformationCB1.1.docx 